\title{
Primate Social Evolution
}

PATRIK LINDENFORS

Stockholm University, Sweden

\section{Primate sociality}

Social evolution concerns questions of sociality and its causes. Central issues include why individuals in some species live in large groups while others live alone, why group composition varies, who moves between groups, and to what degree group living either is just loose association with other individuals or involves complex social interactions. Basic social organization can be described by specifying size, sexual composition, and fission/fusion dynamics of social units. Males and females either live alone or together, in this way yielding five basic potential types of social unit: solitary, monogamous, unimale-multifemale, unifemale-multimale, and multimale-multifemale. Note that social systems are distinct from mating systems, which instead describe general mating practices of individuals, often but not necessarily aligned with how individuals socialize.

Primates display a magnificent variety of types of social organization, ranging from nocturnal and solitary lorises and tarsiers in the tropical forests of Africa and Asia, through pair-bonding gibbons, sakis, and titis, through group-living baboons, macaques, and vervet monkeys, culminating numerically in the nightly reaggregating sleeping herds of gelada baboons in Ethiopia, which consist of many independent stable reproductive units containing one or several individuals of each sex. In contrast to most other mammals, the majority of primate species have social groups composed of both males and females. Note that social primates do not just live in loose associations of independent individuals; instead, each individual is a member of an intricate network of socially interacting individuals that uses various strategies for survival and reproduction. Social relationships with other members of these social units involve kinship, dominance, and short- and long-term coalitions, where each individual has a specific role depending on who is being interacted with and where these roles change over the life cycle. Even solitary primates, though foraging alone, have social interactions with their neighbors and differing degrees of overlapping ranges. In some "solitary" nocturnal species, individuals spend the day together in sleeping groups, and males and females sometimes defend a territory together (Kappeler and Van Schaik 2002).

Phylogenetic analyses indicate a single initial shift from an ancestral solitary lifestyle to a multimale-multifemale type of sociality in the anthropoid ancestral line, sometime after anthropoid primates split from strepsirhines and tarsiers. Most strepsirhines and tarsiers have remained nocturnal and solitary, the main exception being the lemurs of Madagascar, where another shift to sociality has resulted in many species being diurnal

The International Encyclopedia of Anthropology. Edited by Hilary Callan.

(c) 2018 John Wiley \& Sons, Ltd. Published 2018 by John Wiley \& Sons, Ltd.

DOI: 10.1002/9781118924396.wbiea1720 
and multimale. The greatest variation in sociality, however, is found in the anthropoid lineage, which includes solitary, multimale, unimale, and monogamous species. In the hominid line alone, we can find monogamous gibbons, solitary orangutans, unimale-multifemale gorillas, and multimale-multifemale chimpanzees, bonobos, and humans.

Since primates started out as solitary foragers, this multitude of immensely complex social systems stems entirely from derived traits (Shultz, Opie, and Atkinson 2011). Reviewed here are proposed explanations of how this diversity of social systems evolved and how we can understand why individuals of some species live in groups while others do not, why group size and composition vary, and why some individuals stay in their natal groups while others move. The answers are not completely straightforward, especially since new critical examinations of traditional explanations have indicated that we do not know the answers to these questions anywhere near as well as we thought we did (Clutton-Brock and Janson 2012; Thierry 2008).

It also should be noted that sociality is not necessarily a fixed trait but that many primate species display flexible social organizations, where the degree of flexibility is coupled with sociality itself. For example, species living in larger groups tend to have more flexible social organization, probably because of the relatively lower cost of adding or subtracting group members. Also, higher social flexibility is correlated with cognitive ability, as indicated by a larger neocortex in relation to the rest of the brain. Variation in social systems can thus be observed not only within species but also within populations. Hence, social organization is not always the result of long-term evolutionary selection processes but can also be a rapid response to varying ecological circumstances (Kamilar and Baden 2014).

\section{The socioecological model of social evolution}

The most commonly invoked explanatory model of primate social evolution is termed the "socioecological model" of social evolution, where social systems emerge from behaviors caused by individuals' responses to risks and resources in the environment. However, sociality itself introduces new constraints on the options of these individuals, resulting in a feedback loop where the degree and form of sociality itself reaffect the degree and form of sociality.

The socioecological model of social evolution is based on the deduction that since female fitness is mainly determined by ecological factors, such as securing resources and protection from predation and overenthusiastic males, female sociality should be understandable through knowledge of females' ecological settings. Males, on the other hand, can reach higher levels of fitness benefits through mating with several females. Thus, male sociality should be understandable mainly through knowledge of female spatiotemporal spacing (Kappeler and Van Schaik 2002).

Kin-selection theory leads to the general prediction that sociality among kin should be more common than sociality among non-kin. This prediction is borne out in that most primate social groups consist of genetically related females, with several exceptions, for example in the Hominidae, where it is generally females, not males, 
that migrate to other groups, a behavior resulting in social groups composed mainly of genetically related males and immigrant females. However, some primate social groups consists of several more or less unrelated clans of related females living together. Note also that the socioecological model does not include explanations of phylogenetic inertia and historical constraints-the observation that species are more like closely related species than would be expected from ecology alone (Shultz, Opie, and Atkinson 2011). The explanation for this latter pattern comes down to genetic inertia-evolution takes time.

Historically, the first suggestion for which factor best explains female gregariousness was the degree to which food resources are clumped. Later, predation was introduced as another important factor and, over time, further refinements to the model have been made, adding a number of factors that are sometimes both causes and consequences of sociality, as summarized in Table 1 . The major important factors affecting sociality are now believed to be predation risk, food distribution and population density, main type of competition (within the social group or between social groups), presence of infanticide, dominance hierarchies, frequency of coalition formation, presence of nepotism, and which sex disperses. However, some components of this contemporary complex socioecological model are better backed up by empirical evidence than others.

In the extended socioecological model, a patchy distribution of resources should result in competition between group members, which should in turn develop into dominance hierarchies and long-term alliances with relatives as well as mutualistic coalitions with nonrelatives. Alternatively, group living to gain protection from predation introduces the possibility of monopolizing resources. Cooperation to collectively defend resources should in turn produce stable, linear hierarchies, particularly among relatives, who will tend to serve their genetic self-interest. Intergroup competition should also result in an increased cost of dispersal for females, as any dispersing female would lose her supportive social network (and thus have a reduced ability to claim resources) and temporarily risk predation. These processes together result in territorial philopatric societies of nepotistically cooperating female kin ("resident-nepotistic" in Table 1).

A general point needs to be made here concerning hierarchical social groups: Why don't low-ranking females just leave? The explanation is that social groups outcompete individuals when there is competition over access to patchy food; in competition over food patches, it may be necessary to be a member of a group. Put differently, low-ranking individuals should stay when the costs associated with feeding as a low-ranking member of a group are lower than the costs associated with feeding alone; it is better to be in a group and get a little than be alone and get nothing. If the costs of belonging to a group become larger than the costs of going at it alone (e.g., if food patches are too small or exclusion of lower-ranking individuals is too effective), lower-ranked individuals are predicted to leave (or form coalitions). Members of a social unit therefore tend to disperse when group size is larger than average, but it has also been shown that individuals in fission/fusion societies disperse if competition becomes too intense within their social group. Such evidence provides support for the idea that food competition puts a ceiling on the number of members of social groups. 


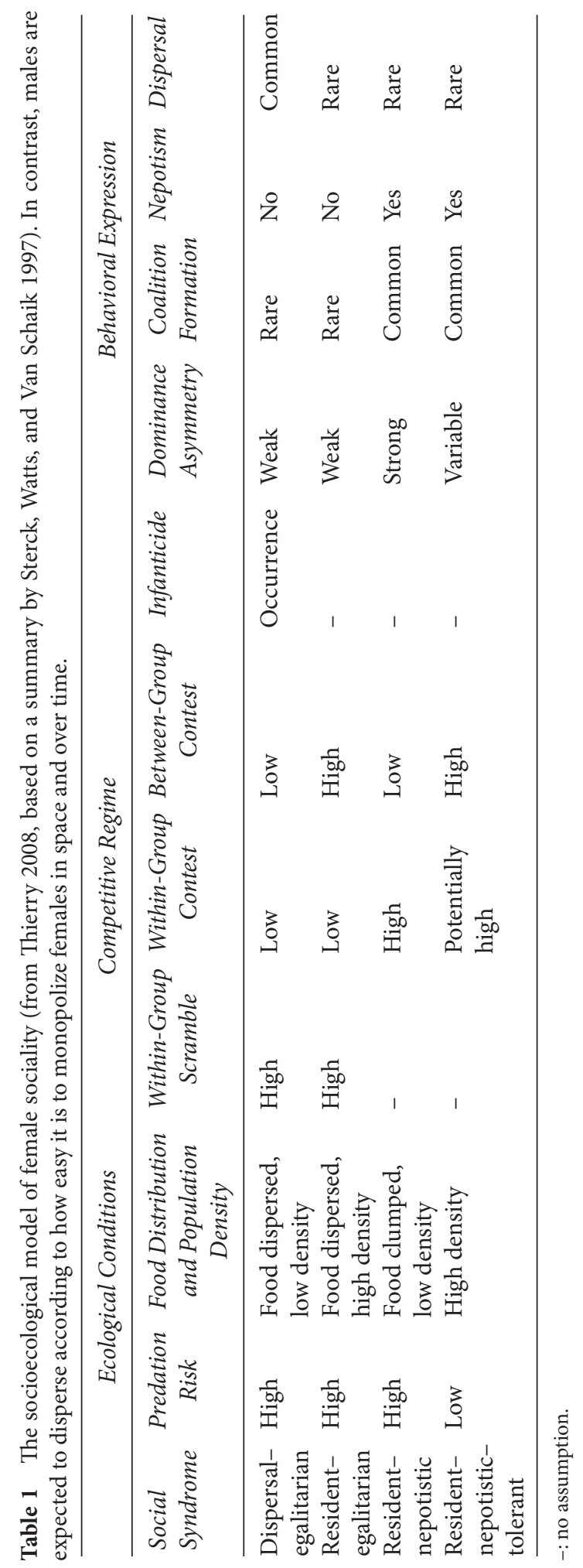


If ecological circumstances do not allow for monopolization of food resources, competition within groups is expected to be lower. Species with low within-group competition will, according to the model, not develop any strong dominance hierarchies and thus coalition forming will be of less importance. In this type of group, it is easier for females to move in and out of social contexts, making social bonds weaker but also making competition less important ("dispersal-egalitarian" in Table 1).

However, if ecological circumstances are such that strong competition between groups for patchy resources becomes important, such competition can favor female philopatry, even if competition is weak within groups. Again, belonging to a group that has secured resources is important in preventing female dispersal. This scenario also leads to sociality, but of a less internally competitive kind ("resident-egalitarian" in Table 1). Note that it is possible to deceitfully withhold support in conflicts with other social units, a fact that leads to the prediction that females in this type of group should prefer to bond with relatives. Since competition between groups is expected to be of less importance than competition within groups, resident-nepotistic societies are expected to be more common than resident-egalitarian societies.

The remaining category ("resident-nepotistic-tolerant" in Table 1) is hypothesized to be the result of an interaction between high within- and between-group competition, where high within-group competition results in stable dominance hierarchies but where the exclusion of subordinates is kept in check by the need for coalition partners in between-group competition. Group members here stick together in family groups but are expected to be more tolerant of other group members than in resident-nepotistic groups.

Most extant primate species belong to either resident-nepotistic or dispersalegalitarian types of social groups (Sterck, Watts, and Van Schaik 1997). Note that the complex interactions between food patchiness, predation risk, and group size make the socioecological model difficult to test empirically. It is particularly hard to estimate relevant patch size and distribution. Also, as already pointed out, closely related species have more similar social systems than would be expected from their feeding ecology alone, which indicates that there are further factors in play (e.g., aspects of inheritance that are revealed through phylogenetic signals). Note also that there is no real explanation in the model of why some species are monogamous. Current opinions among researchers on the merits of the socioecological model are therefore divided (Clutton-Brock and Janson 2012; Thierry 2008).

\section{Four key assumptions underlying the socioecological model}

There are four key assumptions underlying the socioecological model: first, between-group competition, resource patchiness, and predation favor the evolution of sociality in females, with group size being constrained by the resulting within-group feeding competition; second, increased within-group competition and resulting dominance hierarchies mean that the variability of female fitness increases as some females can monopolize more resources than others; third, females will remain in their natal group when feeding competition is intense and will disperse when food 
is more evenly dispersed; and fourth, males will distribute themselves depending on how females are dispersed. These four assumptions will be examined in turn.

\section{Predation, contest competition, and scramble competition}

Sociality reduces predation risk, an observation that is not only valid for primates but also for animals in general. Through numbers alone, when a social unit is larger, this means that a higher number of vigilant individuals are available to detect predators, which frees up each individual to forage more and look for predators less. Further, carnivores have been shown to have lower success rates when hunting members of social units than when hunting lone prey, due in part to dilution and selfish-herd effects, but there is also occasional joint defense against predators. Consequently, diurnal primates living under high predation pressure tend to live in groups. Living in large groups also makes coalition formation possible. Larger groups can exclude smaller groups from resources through contest competition, if food patches are clumped and possible for groups to monopolize. It has also been observed that larger groups sometimes attack and even exterminate smaller groups. Additionally, larger groups of emigrants succeed in establishing new groups more often than do smaller groups of emigrants. Thus, there are several measurable benefits of belonging to larger groups rather than smaller ones, concerning both predation and resource defense. This is countered, however, by competition between individuals within the social unit for food resources through scramble competition. There are thus three selection forces operating simultaneously that govern group size and the nature of the social interactions within the group: protection from predation, intergroup feeding competition, and intragroup competition for food patches. Quantifying these factors in relation to each other, and determining relevant measures of the underlying ecological factors, is a difficult task, a problem that makes the socioecological model of social evolution challenging to test empirically (Clutton-Brock and Janson 2012; Thierry 2008).

\section{Variability in female fitness}

Within-group competition over resources increases with group size as a larger number of group members means that there are more individuals competing for the same food resources. Consequentially, members of larger groups are more stressed than members of smaller groups. The intensity of within-group competition over food varies with the quality, quantity, and dispersal of food resources. There exist several studies indicating that food distribution affects the number of competitive encounters between group members and specifying how social relationships and the ability to form coalitions affect fitness. There are also a number of studies reporting variability in nutrient intake between individuals resulting from increased competition over food. However, there is as yet no consistent result across a large number of populations that validates the hypothetical correlation between social structure and the degree of feeding competition-instead, similar social systems are commonly found in closely related species even if these have different feeding ecologies. Also, 
there is no large-scale study that has investigated whether the degree of reproductive skew is related to differences in dietary intake (Clutton-Brock and Janson 2012).

\section{Dispersal}

Dispersal can be described as either male or female philopatry, depending on the sex of the individuals that remain with their natal group. In species where both sexes are reported to disperse, it is usually more common for one sex to move away than the other. In most primates, as in most other social mammals, males disperse after reaching sexual maturity while females stay in their social groups. Exceptions to this rule are not rare in primates, being found in many New World species, such as howler monkeys, marikis, spider monkeys, and tamarins, as well as Old World species such as bonobos, chimpanzees, gorillas, hamadryas baboons, and red colobus monkeys. In some of these species, females sometimes migrate together or serially to the same recipient group, meaning that groups can end up with related females even in male philopatric species. In species with female philopatry, males living together generally have neutral or antagonistic relationships while females develop closer social bonds with other females; in species with male philopatry, the opposite is true. It has been suggested that female migration may be explained by inbreeding avoidance, where females disperse when average male tenure in a group is longer than the time it takes for females to become sexually mature, while males disperse in all other cases.

\section{Primate males go where the females are}

The number of males in primate social groups is determined by the number of females in the group but also by how synchronously these females breed-that is, it depends on the monopolization potential of male access to female mating partners, either by exclusion of other males or through female choice, depending on the defensibility of females and the requirements of infant care (Kappeler and Van Schaik 2002). Comparative tests across species of primates have shown that the number of males in primate groups correlates with the number of females. As female group size increases due to the ecological benefits of group living, it becomes progressively more difficult for a single male to defend access to a whole group of females, resulting in multimale-multifemale groups. Additional variation in male membership can be accounted for by female mating behavior: species with more synchronously mating females have relatively more males per group. Thus, the number of males in primate groups varies according to the degree of competition over females (sexual selection). Whereas food resources can be shared, fertilizations cannot-either a male succeeds in fertilizing a female or not. Therefore, male relationships are typically more competitive and intolerant and result in more clear-cut dominance hierarchies, and this competition has left physical imprints such as sexual dimorphism in size and canines (Thorén, Lindenfors, and Kappeler 2006). However, alliances and affiliative behaviors are nevertheless observed between 
males in some social groups. In the few cases where males are philopatric and females migrate (e.g., chimpanzees), coalitionary behavior between males is well developed.

\section{Three further factors believed to influence sociality}

Three other factors believed to influence social life are infanticide avoidance, intersexual sociality, and cognitive capacity. These will be examined in turn.

\section{Infanticide and monogamy}

It is known from many mammal species that males who take over the dominant position in a social unit will often try to kill youngsters fathered by the previous dominant male, a behavior fundamentally detrimental to the afflicted female's fitness. Consequently, there are many papers arguing for and testing the hypothesis that primate social organization not only is determined by ecological factors but also is shaped by females striving to ensure protection from infanticide. Since there is a low infanticide rate in monogamous species, it has been argued that monogamy may have evolved as a response to infanticide. As has been pointed out, however, it is only when the duration of lactation exceeds that of gestation (which is uncommon among monogamous species, except in hominins) that it is beneficial to kill other males' offspring. An alternative hypothesis is instead that monogamy is a means to monopolize the reproduction of single females, when females have been driven apart by ecological circumstances. To defend and provision common offspring is a logical extension of such mate guarding. Weighing the evidence indicates that the latter hypothesis has stronger empirical backing, even if more research is necessary to settle the issue (De Waal and Gavrilets 2013).

\section{Intersexual sociality}

Relationships between primate males and females are ultimately shaped by differences in what limits fitness in each sex and the ensuing sexual conflicts and sexual selection. Adult males are normally dominant over adult females, except in most lemurs, a factor that can lead to sexual coercion and in extreme cases infanticide (see previous section). Variables that account for this asymmetry are that males are larger and have larger canines than females in many species, a pattern itself the consequence of differences in social structure between males and females, where males are selected to be larger through intramale competition over mating opportunities (Thorén, Lindenfors, and Kappeler 2006). Females have a number of counterstrategies to deal with coercive behavior, including alliances with other females and seeking protection from other males. In cases where there are affiliative bonds between males and females, females groom males more than males groom females, while males provide protection from other males and predators. 


\section{The Machiavellian intelligence (or social brain) hypothesis}

The Machiavellian intelligence hypothesis (Byrne and Whiten 1988) states that the degree of social complexity should be of importance for brain evolution, since keeping track of a larger number of interactions in complex social networks places higher demands on individual cognition. As a consequence, social complexity should select for larger neocortices. Conversely, neocortex size has been hypothesized to place a limit on the number of social interactions an individual can keep track of and thus to place a limit on group size (e.g., Shultz et al. 2014). This hypothesis has even been extended to propose a limit on the number of individual in groups that the human mind can typically handle ("Dunbar's number": about 150). However, the general reasoning behind this number depends crucially on the assumption that primate social units are uniquely limited by species' cognitive abilities. As we have seen, there are a number of other factors that also limit social unit size, meaning that a relationship between cognitive capacity and social group size would systematically underestimate maximum social group size. Further, the differences between group sizes of chimpanzees and human hunter-gatherers are not predicted by the theory-while sleeping camps may be small, social networks may extend to several hundreds. Other empirical studies of human networks have indicated that human social groups in general come in a large variety of sizes, many much larger than Dunbar's number. Although the relationship between primate group sizes and the relative size of the neocortex has been replicated in many studies, the relationship is only empirically verified for female group size, not male group size (Lindenfors, Nunn, and Barton 2007). Further, studies of nonprimate mammals have shown that monogamous species have the largest neocortices in relation to the rest of the brain, indicating that another process may be operating. However, proponents of the social brain hypothesis have argued that it may be more cognitively demanding to maintain stable pair bonds than to interact socially with many individuals, except in anthropoid primates (Shultz et al. 2014). The observed exception, that there is a correlation in anthropoid primates between group size and relative neocortex size, is instead explained through the hypothesis that primates have extended their pair-bond-like relationships to other group members, thus creating the quantitative relationship observed between the relative size of the neocortex and group size, in effect having developed friendships-pair-bond-like relationships without the sexual connotations-between all members of the social group (Shultz et al. 2014). Whether this is an ad hoc hypothesis or an empirically verifiable pattern remains to be tested.

SEE ALSO: Apes, Origins of; Behavioral Ecology, Primate; Biological and Evolutionary Anthropology; Cooperation, Evolution of; Empathy, Evolution of; Goodall, Jane (b. 1934); Humor and Laughter; Imitation, Social Learning, and Cultural Traditions; Inbreeding Avoidance, Evolutionary Mechanisms of; Incest, Theoretical Perspectives on; Intelligence, Evolution of; Intersubjectivity and Joint Attention; Kinship (Early Human), the Archaeological Evidence for; McLennan, John Ferguson (1827-81); Pair Bonds and the Evolution of Monogamy; Play; Primate Intentional Communication; Sexual Conflict Theory; Sexual Dimorphism in Hominin Ancestors; Social Brain 
Hypothesis; Social Network Analysis; Theory of Mind in Primates and Humans, Comparative Evolution of

\section{REFERENCES AND FURTHER READING}

Byrne, Richard, and Andrew Whiten, eds. 1988. Machiavellian Intelligence. Oxford: Oxford University Press.

Clutton-Brock, Tim, and Charles Janson. 2012. "Primate Socioecology at the Crossroads: Past, Present, and Future.” Evolutionary Anthropology: Issues, News, and Reviews 21: 136-50. doi:10.1002/evan.21316.

De Waal, Frans B. M., and Sergey Gavrilets. 2013. "Monogamy with a Purpose." Proceedings of the National Academy of Sciences of the United States of America 110: 15167-68. doi:10.1073/pnas.1315839110.

Kamilar, Jason M., and Andrea L. Baden. 2014. "What Drives Flexibility in Primate Social Organization?” Behavioral Ecology and Sociobiology 68: 1677-92. doi:10.1007/s00265-014-1776-x.

Kappeler, Peter M., and Carel P. van Schaik. 2002. "Evolution of Primate Social Systems." International Journal of Primatology 23: 707-40. doi:10.1023/A:1015520830318.

Lindenfors, Patrik, Charles L. Nunn, and Robert Barton. 2007. "Primate Brain Architecture and Selection in Relation to Sex.” BMC Biology 5: 20. doi:10.1186/1741-7007-5-20.

Mitani, John C., Josep Call, Peter M. Kappeler, Ryne A. Palombit, and Joan B. Silk, eds. 2012. The Evolution of Primate Societies. Chicago: University of Chicago Press.

Shultz, Susanne, Christopher Opie, and Q. D. Atkinson. 2011. "Stepwise Evolution of Stable Sociality in Primates." Nature 479 (7372): 219-22.

Shultz, Susanne, Christopher Opie, Emma Nelson, Q. D. Atkinson, and R. I. M. Dunbar. 2014. "Evolution of Primate Social Systems: Implications for Hominin Social Evolution." In Lucy to Language: The Benchmark Papers, edited by R. I. M. Dunbar, Clive Gamble, and J. A. J. Gowlett, 317-32. Oxford: Oxford University Press.

Smuts, Barbara B., Dorothy L. Cheney, Robert M. Seyfarth, Richard W. Wrangham, and Thomas T. Struhsaker. 1987. Primate Societies. Chicago: University of Chicago Press.

Sterck, Elisabeth H. M., David P. Watts, and Carel P. van Schaik. 1997. "The Evolution of Female Social Relationships in Nonhuman Primates." Behavioral Ecology and Sociobiology 41: 291-309. doi:10.1007/s002650050390.

Thierry, Bernard. 2008. "Primate Socioecology, the Lost Dream of Ecological Determinism." Evolutionary Anthropology: Issues, News, and Reviews 17: 93-96. doi:10.1002/evan.20168.

Thorén, Sandra, Patrik Lindenfors, and Peter M. Kappeler. 2006. "Phylogenetic Analyses of Dimorphism in Primates: Evidence for Stronger Selection on Canine Size than on Body Size." American Journal of Physical Anthropology 130: 50-59. doi:10.1002/ajpa.20321. 